\title{
Atividades escolares presenciais na sindemia de covid-19: razões para comemorar?
}

\section{Face-to-face class activities during the covid-19 syndemic:}

\section{reasons to celebrate?}

\author{
Eduardo Rumenig ${ }^{\mathrm{a}, \mathrm{b}, \mathrm{c}}$ \\ (D) https://orcid.org/0000-0001-7040-6995 \\ E-mail: erumenig®usp.br \\ ${ }^{a}$ Faculdade de Filosofia, Letras e Humanidades, Universidade \\ de São Paulo, São Paulo, SP, Brasil. \\ buniversidade de São Paulo. Escola de Educação Física e Esporte. \\ São Paulo, SP, Brasil. \\ 'Cebrap, São Paulo, SP, Brasil.
}

\section{Correspondência}

Escola de Educação Física e Esporte. Av. Professor Mello Moraes, 65, Butantã, São Paulo, SP, Brasil. CEP 05508-030.

\section{Resumo}

Sindemia é o termo utilizado para designar como interações biológicas e sociais influenciam no comportamento de uma determinada doença. A retomada das atividades escolares presenciais em meio à sindemia de covid-19 suscita controvérsias. Os defensores alegam que o retorno presencial mitigaria vulnerabilidades sociais, sanitárias e educacionais, enquanto os críticos afirmam que tal decisão contribuiria para a disseminação do vírus. O presente estudo analisa a dinâmica das internações e mortes por covid-19 entre estudantes da educação básica mediante a retomada das atividades escolares presenciais no município de São Paulo. Ademais, procura desvelar se a incidência de infecção por Sars-Cov-2 entre profissionais da educação teria relação com a qualidade socioterritorial que circunscreve as unidades de ensino básico (UE). As análises foram realizadas utilizando base de dados de instituições públicas paulistas. A retomada das atividades presenciais coincidiu com o aumento de internações e óbitos por covid-19 entre estudantes. O pico ocorreu 15 dias após o início das aulas. As UE inseridas em territórios com menores índices de desenvolvimento humano (IDH) registraram as maiores taxas de infecção por Sars-Cov-2. Retomar as aulas presenciais sem diminuir os riscos de contaminação parece ameaçar principalmente os indivíduos de territórios vulneráveis.

Palavras-chave: Educação; Covid-19; Desigualdades Sociais; Sindemia. 


\section{Abstract}

The term syndemic has been used to designate how biological and social interactions influence the behavior of a particular disease. The resumption of face-to-face school activities amidst the covid-19 syndemic sparks considerable controversy: while supporters claim that returning to school would mitigate social, health, and educational vulnerabilities, critics say that such a decision would help to spread the virus. In this scenario, this study analyzes the dynamics of hospital admissions and deaths related to covid-19 among elementary students in terms of the resumption of face-to-face school activities in the city of São Paulo, Brazil. Moreover, it also investigates the association between the incidence of covid-19 among education professionals and the sociospacial properties in which the based education units (UEs) are located. The analyses were conducted with data from the public institutions of São Paulo. The results indicate that in-person activities resumption coincided with an increase in admissions and deaths from covid-19 among students, peaking 15 days after the beginning of the classes. UEs located in territories with lower human development indices registered the highest SARS-Cov-2 infection rates. Thus, resuming inperson classes without mitigating contamination risks poses a threat for the population, especially for those from vulnerable territories.

Keywords: Education; Covid-19; Social Inequalities; Syndemic.

\section{Introdução}

No início de 2021, os poderes executivos estadual e municipal de São Paulo decretaram o retorno às aulas presenciais (São Paulo, 2020). 0 anúncio, feito em 13 de janeiro numa coletiva de imprensa (Martins; Bragança; Brito, 2021), alegava que o retorno escolar era imprescindível para atenuar as desigualdades socioeconômicas e educacionais agravadas pela sindemia de Sars-Cov-2 (Virginio, 2021). Diante do desemprego, do subemprego (IBGE, 2021), da perda de renda, do déficit de aprendizagem, bem como da elevação do custo de vida (Diese, 2020), prefeitos e governadores reconheceram a necessidade de retomar as aulas a fim de atenuar as agruras das famílias, sobretudo as mais pobres.

Figurava ainda o argumento de que as crianças e jovens seriam menos reativos ao Sars-Cov-2 (Ludvigsson, 2020), e que com a readequação das unidades de ensino básico (UE) aos protocolos sanitários, seria possível mitigar eventuais externalidades negativas sanitárias deflagradas pela sua reabertura (Unicef, 2020). Alegava-se, portanto, que o retorno às aulas presenciais atenuaria as vulnerabilidades socioeconômicas e educacionais sem concomitante elevação no número de infecções por covid-19.

Críticos, todavia, alertavam que o retorno às UE aumentaria os deslocamentos urbanos cotidianos, elevando o risco de contaminação (IbarraEspinosa et al., 2021). Em São Paulo, por exemplo, $35 \%$ das viagens cotidianas (14,6 milhões de viagens) são motivadas por objetivos educacionais, sendo $59 \%$ desse contingente por transporte coletivo (CMSP, 2019). Mesmo restringindo a presença de crianças e jovens no espaço escolar, haveria aglomeração nos transportes de massa. E ainda que a frequência dos estudantes fosse reduzida a 35\%, como propunham prefeitos e governadores, o ensino presencial colocaria ao menos 5 milhões de pessoas em circulação diariamente pela cidade (CMSP, 2019).

Entidades sindicais alegavam ainda que boa parte das escolas não dispunham de recursos materiais, equipes capacitadas e infraestrutura adequada para receber estudantes e profissionais de ensino (Figueiredo; Ianelli, 2021). Criticavam os protocolos 
sanitários, argumentando que não contemplavam a complexidade da rotina escolar (Comissão de Biossegurança do CCAE, 2020), e que a redução orçamentária dos investimentos em educação a menor da década (Todos Pela Educação, 2021) - e a morosidade das políticas públicas educacionais impossibilitariam a adequação das escolas aos protocolos sanitários.

A qualidade dos equipamentos de proteção individual fornecidos pelos poderes públicos estadual e municipal e a ausência de assistência hospitalar eram agravantes que, segundo os sindicatos, desencorajariam o ensino presencial. Em seu lugar, sugeriam a implementação de programas de segurança alimentar, assistência social e pedagógica como forma de assegurar a aprendizagem e condições dignas de existência sem prescindir do distanciamento físico, imprescindível para conter a disseminação do vírus (Van Lancker; Parolin, 2020).

Até então não havia evidências empíricas que pudessem avaliar os efeitos das atividades educacionais presenciais no comportamento do Sars-Cov-2. O cenário mudou em fevereiro de 2021, quando a maior parte das escolas básicas adotaram o ensino presencial, recebendo até 35\% dos estudantes em caráter não-obrigatório, e a quase totalidade dos profissionais de educação (São Paulo, 2021). Utilizo esses dois meses de retorno escolar, compreendidos entre fevereiro e março de 2021, para analisar o comportamento das internações e óbitos deflagrados por covid-19 entre crianças e jovens em idade escolar do município de São Paulo. O intervalo temporal antecede o retorno presencial em sete dias, ocorrido em 8 de fevereiro de 2021, e sucede em 13 dias a medida que interrompeu as aulas, em 17 de março de 2021.

Adicionalmente, discrimino as UE da rede pública com maior incidência de contágio por covid-19 entre os profissionais de educação. Conforme suscitado por alguns autores (Horton, 2020; Kirby, 2020), territórios e populações mais vulneráveis seriam mais suscetíveis ao SarsCov-2. Não por acaso, Horton (2020) adota o termo sindemia para caracterizar o cenário deflagrado pela covid-19, trazendo a lume as interações biológicas e sociais que contribuem para a propagação e controle do vírus. Por conseguinte, desigualdades socioeconômicas deveriam ser consideradas no prognóstico, tratamento e desenvolvimento de políticas públicas educacionais e sanitárias.

É provável, portanto, que as vulnerabilidades socioterritoriais que influenciam na disseminação da covid-19 também se reproduzam no âmbito escolar, afetando principalmente estudantes e profissionais da educação que atuam em equipamentos situados em territórios com menor qualidade socioespacial.

Para apreender essas questões, dividi o artigo em cinco seções além da introdução (primeira seção). Na segunda seção, esclareço a metodologia de obtenção e análise dos dados relativos às internações e mortes por covid-19 entre escolares no município de São Paulo, bem como o processo de geocodificação das UE e dos respectivos territórios que circundam esses equipamentos públicos. Na terceira seção descrevo os resultados, seguidos de uma discussão. Na quarta há uma reflexão sobre as prováveis relações entre atividades educacionais presenciais e a sindemia do covid-19. Encerro o texto com algumas considerações finais.

\section{Metodologia}

Uma breve contextualização do município de São Paulo, das escolas de nível básico sediadas na municipalidade, bem como o metabolismo urbano associado ao funcionamento dessas instituições de ensino antecedem os detalhes metodológicos.

\section{Rede de ensino básico em São Paulo}

O município de São Paulo compreende uma área de $1.509 \mathrm{~km}^{2}$ ocupada por aproximadamente 13 milhões de pessoas ( $7.398 \mathrm{hab} . / \mathrm{km}^{2}$ ). Nesse território estão distribuídas 9.168 UE, sendo 4.788 unidades de ensino infantil, 2.997 de ensino fundamental e 1.383 de ensino médio, públicas e privadas (IBGE, 2021). Aproximadamente $52 \mathrm{mil}$ professores atuam na rede infantil, enquanto as redes de ensino fundamental (69.443) e médio (27.649) contam com aproximadamente $100 \mathrm{mil}$ professores, que atendem aproximadamente 2,5 milhões de crianças e jovens diariamente, sendo 
662 mil na educação infantil, 1,4 milhão no ensino fundamental e 420 mil no ensino médio (IBGE, 2021).

O último censo escolar (INEP, 2020) de 2020 revela que as UE do município de São Paulo apresentam infraestrutura semelhante, com serviços de água encanada e energia elétrica (99\%), coleta de lixo periódica (100\%) e rede de esgoto (96\%) quase universais. Além disso, a maioria dispõe de cozinha e sanitários (99\%), embora espaços como bibliotecas, salas de leitura, quadras ou espaços de lazer, laboratórios de informática e ciências sejam menos frequentes. Somente $9 \%$ das escolas públicas de educação infantil (o a 5 anos) contam com bibliotecas, 21\% contam com quadras ou áreas de lazer, $18 \%$ possuem laboratórios de informática e nenhuma possui laboratório de ciências. As escolas de ensino fundamental, por sua vez, apresentam maior oferta de bibliotecas (14\%), quadras ou espaços de lazer ( $84 \%$ ), laboratório de informática (78\%) e de ciências (18\%). Algumas escolas de ensino médio também contam com bibliotecas (10\%), embora em menor número que as escolas de ensino fundamental. Quase todas possuem quadras e laboratórios de informática (94\%), mas somente $18 \%$ contam com laboratório de ciências.

Os territórios que circunscrevem essas UE também apresentam distinções, principalmente em termos de oferta de oportunidades como trabalho, equipamentos e serviços públicos (Hochman et al., 2007). Embora serviços públicos como coleta seletiva, água potável e energia elétrica sejam quase universalmente distribuídos, outros como oportunidades de emprego, serviços de saúde e assistência ainda são centralizados, compelindo seus habitantes a realizarem deslocamentos diários, quase sempre em condições precárias de transporte (Hochman et al., 2007; Marques, 2015). Quanto menor a renda, maiores as distâncias e a duração das viagens (Giannotti et al., 2021).

Aproximadamente 14 milhões de viagens cotidianas são motivadas por razões educacionais, sendo $59 \%$ por transporte coletivo (CMSP, 2019). Embora a legislação determine um raio de $2 \mathrm{~km}$ entre o endereço da UE e o local de residência do estudante (SME, 2021), reduzindo a necessidade do transporte motorizado entre os mais jovens, para os profissionais de educação não há nenhuma normativa que limite o local de trabalho em relação a residência; o que implica em milhares de viagens, eventualmente por transportes públicos, cujas condições podem ensejar riscos à saúde devido a aglomerações em meio à sindemia de covid-19.

Não obstante, quase 100 mil crianças e jovens utilizam transporte coletivo escolar (como microônibus) para acessar as UE, promovendo riscos sanitários, seja para os estudantes, seja para a bolha social que circunscreve esses jovens, uma vez que esse tipo de transporte também pode engendrar aglomerações. Assim, o ensino presencial não interfere apenas na dinâmica escolar, mas no próprio metabolismo urbano, com graves implicações para o controle da sindemia. Nossa tarefa foi observar o comportamento das internações e mortes mediante o retorno presencial às escolas, considerando os territórios nos quais essas UE se inserem.

\section{Fonte de dados}

As informações das internações e óbitos por covid-19 foram retiradas do Sistema de Monitoramento Inteligente (SIMI) ${ }^{1}$ do governo do estado de São Paulo. Em meados de 2020 o governo estadual criou um comitê de crise para monitorar e gerenciar a sindemia, bem como auxiliar administradores públicos na tomada de decisões baseadas em evidências. Esse comitê, batizado de centro de contingência da covid-19, reúne fundações, secretarias e autarquias de diferentes áreas do conhecimento (epidemiologia, infectologia, gestão pública, ciência de dados e saúde pública) com o objetivo de sistematizar, produzir e difundir informações sobre a sindemia no estado de São Paulo.

Foram incluídas todas as internações e óbitos por covid-19 de crianças e jovens com idade entre $1 \mathrm{e}$ 18 anos, de ambos os gêneros. 0 período analisado contemplou os meses de fevereiro (que antecede em 7 dias o retorno presencial às UE) e março de 2021,

1 Disponível em: <https://www.saopaulo.sp.gov.br/planosp/simi/dados-abertos/>. Acesso em: 22 mar. 2021. 
que corresponde a 14 dias após a suspensão das atividades presenciais. Considerando que o período de incubação do Sars-Cov-2 varia de 3 a 10 dias (Zhou et al., 2020), o intervalo contemplado permitiu desvelar a influência das atividades escolares nas taxas de internação e morte dos estudantes, segmentadas por nível de ensino: educação infantil (1 a 5 anos), ensino fundamental I (6 a 10 anos), fundamental II (11 a 15) e ensino médio (16 a 18 anos).

Referente ao registro de infecções por covid-19 entre os profissionais das UE, infelizmente não existem dados oficiais disponíveis. A fim de suprir essa ausência, os sindicatos dos profissionais de educação (Sinpeem, 2021) criaram um banco de dados com os registros de contaminação e óbitos por covid-19, discriminado por UE e por função (equipe de apoio, gestores e professores). A base de dados é alimentada pelos próprios profissionais das UE, que reportam aos sindicatos as contaminações por covid-19. Todos os profissionais que atuam nas UE (equipe de apoio, gestores e professores) foram incluídos nas análises, discriminadas por distrito (subprefeitura).

Ademais, o Índice de Desenvolvimento Humano municipal (IDHm) foi utilizado como marcador da qualidade socioterritorial desses distritos. O IDHm considera escolaridade, renda e saúde dos habitantes, e pode ser utilizado como proxy da qualidade socioterritorial. Valores próximos de 1 indicam elevada qualidade socioterritorial, ao passo que valores próximos de zero são associados a áreas precárias, com renda, escolaridade e expectativa de vida inferiores. Todas as informações socioterritoriais procederam da secretaria de Direitos Humanos e Cidadania (SDHM) da prefeitura de São Paulo. ${ }^{2}$

\section{Processamento e análises}

Os dados sobre contágios nos centros de educação infantil (CEI), escolas municipais de educação infantil (EMEI), escolas de ensino fundamental (EMEF) e ensino médio (EM) disponibilizados pelos sindicatos de educação foram transformados em informação geográfica por meio do software BatchGeo. ${ }^{3}$ Então, o arquivo $\mathrm{kml}$ foi posteriormente processado no software QGIS (versão 3.14, Zürich), em conjunto com as informações sobre a qualidade socioespacial (IDHm) dos territórios que circunscrevem essas UE.

As análises descritivas das taxas de internações e óbitos, segmentadas por faixa etária e gênero, bem como as informações geográficas de Sars-Cov-2 entre profissionais da educação discriminadas por UE e IDHm foram processadas por meio do software RStudio (versão 1.1.14). Todos os resultados são descritos na seção seguinte.

\section{Resultados}

Entre fevereiro e março de 2021 foram contabilizadas 9.435 internações e 53 óbitos por covid-19, sendo 61\% dessas internações (5.765) em março, 20 dias após o início das atividades educacionais presenciais. Segmentados por idade, as crianças abaixo de 5 anos representaram $23 \%$ dos ingressos hospitalares; os estudantes do ensino fundamental I e II, entre 6 e 15 anos, 44\%; ao passo que jovens com idade igual ou superior a 16 anos contabilizaram $33 \%$ das internações. Comparativamente no mesmo período, foram internados 103.392 adultos entre $19 \mathrm{e}$ 59 anos, dos quais 1.483 vieram a óbito, a maior parte também em março (62\%).

Desse total, 9,1\% dos casos e $3 \%$ dos óbitos foram de pessoas em idade escolar (entre 1 e 18 anos). A taxa de mortalidade observada entre os escolares (o,01\%) foi inferior à registrada para adultos abaixo de 59 anos (1,44\%), e muito menor que a registrada pelo município (13\%).

Entre fevereiro e março, o número de internações por covid-19 de pessoas em idade escolar apresentou um aumento expressivo. Entre as crianças da educação infantil (o a 5 anos), o aumento foi de 55\% (793 em fevereiro e 1.233 em março). Já os estudantes dos ensinos fundamental I e II evidenciaram um aumento de $67 \%$ e $50 \%$, respectivamente, sendo 569 casos em fevereiro e 950 casos em março para os jovens entre 6 e 10 anos de idade (fundamental I), e 1.078 casos em fevereiro e 1.623 em março para os estudantes do ensino fundamental II (entre 11 e 15 anos).

\footnotetext{
2 Disponível em: <http://dados.prefeitura.sp.gov.br/dataset/indice-de-desenvolvimento-humano-municipal〉. Acesso em: 22 mar. 2021. 3 Maiores detalhes sobre o software BatchGeo, acesse: <https://batchgeo.com/>. Acesso em: 22 mar. 2021.
} 
A faixa etária correspondente ao ensino médio (16 a 18 anos) registrou um aumento de 59\%, com 1.230 internações em fevereiro e 1.959 em março. Comparativamente, na população adulta entre 19 e 59 anos, houve um aumento de $60 \%$ no número de internações (39.664 em fevereiro e 63.728 em março); assemelhando-se aos valores relativos observados nos indivíduos em idade escolar.
O fechamento das escolas em 17 de março de 2021, associado a outras medidas restritivas de circulação na cidade, reduziram em $8 \%$ os ingressos hospitalares entre a primeira e a segunda quinzena de março, tanto de crianças e jovens quanto de adultos abaixo de 59 anos, suscitando que o ensino remoto talvez seja uma medida efetiva para conter as infecções por Sars-Cov-2. Os detalhes das internações são descritos no Gráfico 1.

\section{Gráfico I - Taxa de internações por Sars-Cov-2 entre menores de 18 anos no município de São Paulo}
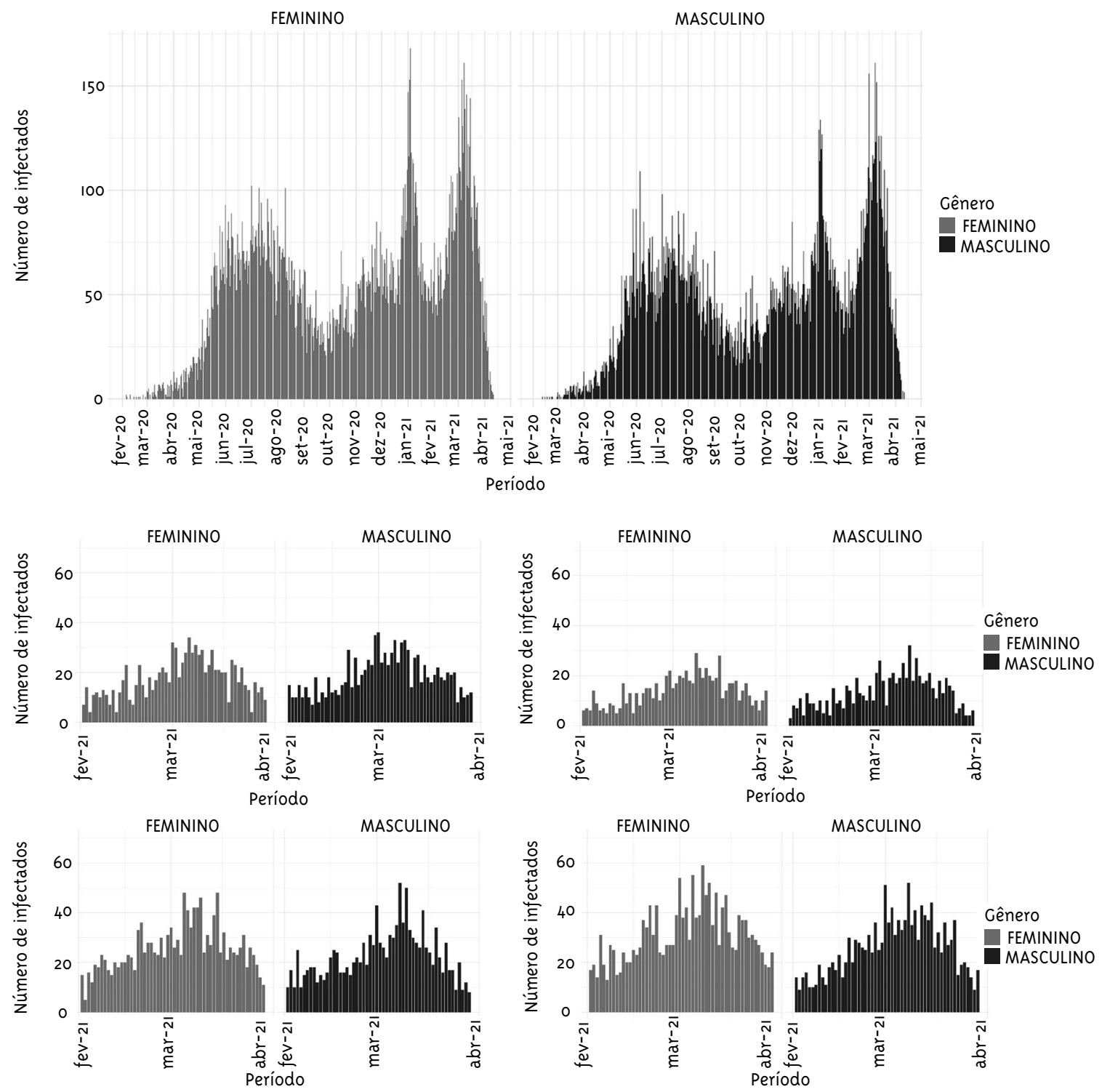

0 gráfico superior discrimina as internações por covid-19 entre menores de 18 anos. Na área central, o gráfico à esquerda discrimina as internações diárias entre escolares da educação infantil, ao passo que a da direita, do fundamental I. Os gráficos inferiores discriminam as internações por covid-ı entre estudantes do ensino fundamental II (à esquerda) e ensino médio (à direita).

Fonte: Elaboração própria a partir dos dados da SIMI. 
No que concerne à incidência de profissionais da educação acometidos por Sars-Cov-2, observa-se que as UE localizadas em áreas de menor qualidade socioterritorial evidenciaram maior incidência de contaminação (Figura 1: mapa à direita). 0 mapa à esquerda discrimina a distribuição das UE da rede pública de ensino (CEU, CEI, EMEI, EMEF e escolas estaduais) no município de São Paulo. Nota-se uma distribuição periférica das UE, o que dificulta discriminar os casos de covid-19 considerando somente o território. Ademais, a heterogeneidade das periferias paulistanas (Marques, 2015) poderia comprometer uma avaliação apenas territorial. Assim, optou-se pelo uso de indicadores socioterritoriais que consideram parâmetros como expectativa de vida, escolaridade e renda para qualificar os territórios. Quanto menor o IDHm do território (área clara), maior a incidência de covid-19 nas UE.

\section{Figura I - Distribuição dos equipamentos educacionais em São Paulo e contaminação por covid-ı entre os profissionais da rede pública de ensino}
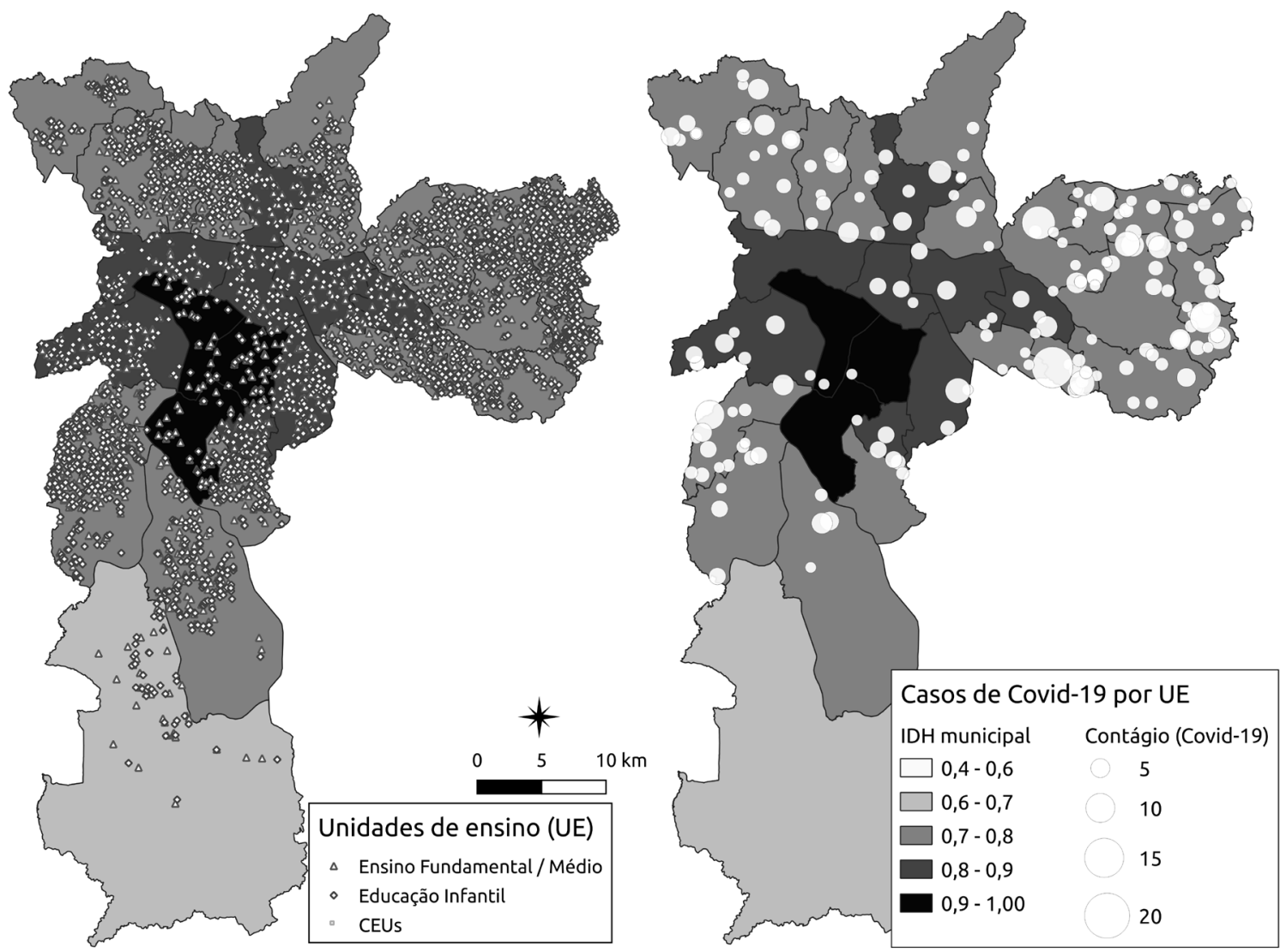

o mapa à esquerda discrimina o número de escolas públicas de educação infantil, ensino fundamental (I e II) e médio. À direita, o número de profissionais acometidos pela covid-19 desde o início da sindemia, segmentado por UE.

Fonte:. Elaboração própria a partir de dados da GeoSampa e Sinpeem.

Os distritos localizados na zona leste da cidade evidenciaram as maiores taxas de Sars-Cov-2 entre os profissionais de educação, com destaque para a Cidade Tiradentes, que coincidentemente registrou um dos menores IDHm do município (o,70 em 2010). Inversamente, o distrito de
Pinheiros (o,94 em 2010) registrou um número insignificante de contágios no mesmo período, evidenciando que quanto menor a qualidade socioterritorial que circunscreve a UE maior o número de profissionais contaminados por covid-19 (Gráfico 2). 


\section{Gráfico 2 - Total de casos de covid-ıg entre profissionais da educação pelo IDHm do distrito}

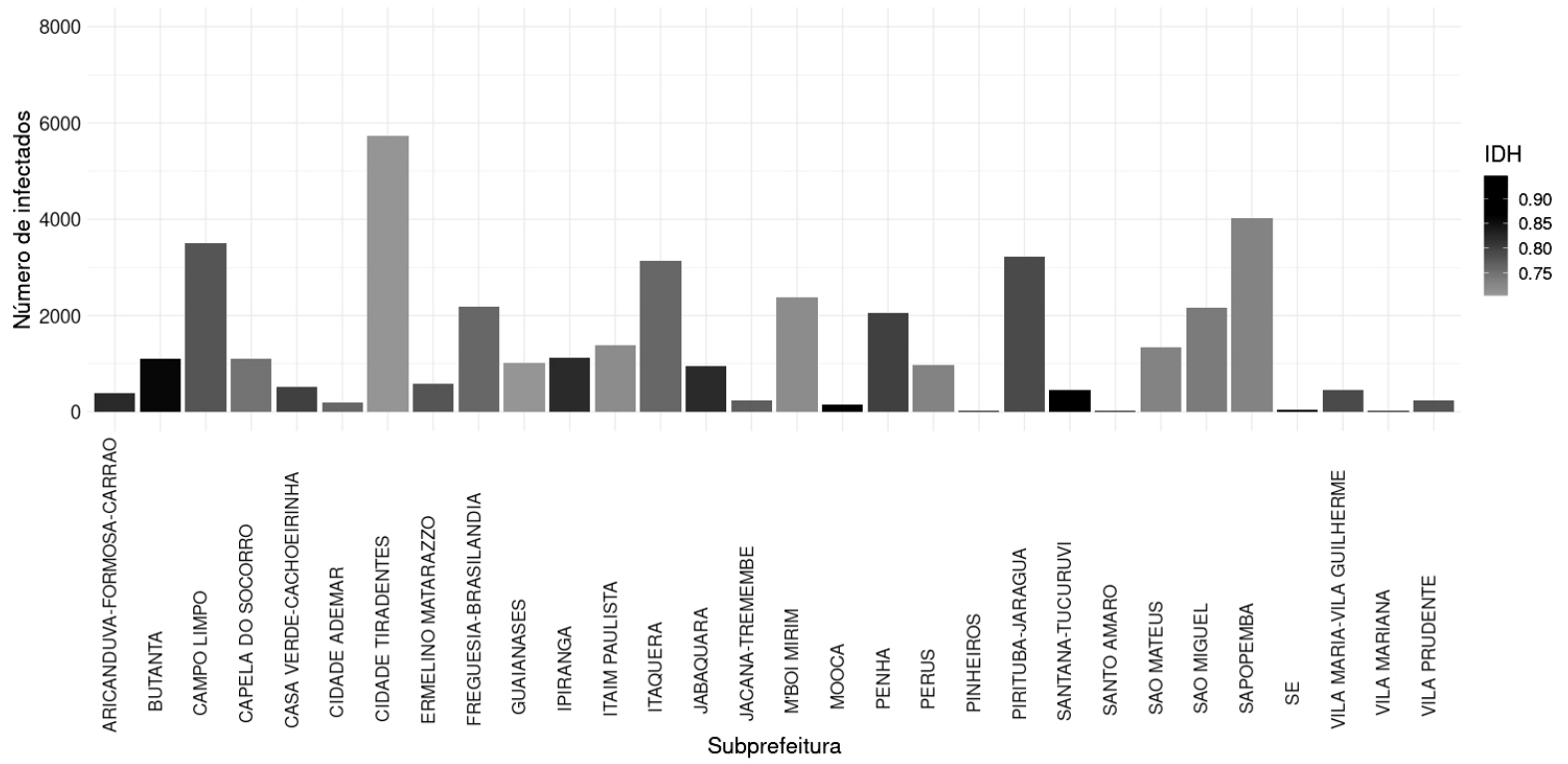

O IDHm pode ser apreendido como proxy da qualidade socioterritorial que circunscreve a escola. Nesse caso, parece existir uma relação inversa entre a incidência de covid-19 e a qualidade socioterritorial.

\section{Discussão}

A proposta do estudo foi analisar a dinâmica das internações e mortes por covid-19 entre crianças e jovens mediante a retomada das atividades escolares presenciais no município de São Paulo, bem como desvelar como a qualidade socioterritorial que circunscreve as UE coincide com os casos de covid-19 entre profissionais da educação básica. A reabertura escolar coincidiu com o aumento das internações e mortes por covid-19 entre pessoas com idade inferior a 18 anos. 0 pico de internações ocorreu 15 dias após o início das aulas presenciais, coincidindo com o período de incubação do vírus, estimado entre 3 e 10 dias (Zhou et al., 2020). Observou-se ainda maior prevalência de casos de covid-19 entre profissionais de educação que atuam em UE inseridas em territórios com menor IDHm, o que mostra que as desigualdades socioterritoriais podem contribuir para maiores taxas de infecção por Sars-Cov-2 também no âmbito escolar.
A retomada das atividades presenciais nas UE levanta controvérsias. Gurdasani et al. (2021), por exemplo, argumentam que reabrir escolas sem mitigar os riscos de contágio contribuiria tanto para o aumento da taxa de transmissibilidade quanto para o surgimento de novas variantes do vírus causador da covid-19. Ademais, o descontrole da sindemia acirraria as desigualdades educacionais já existentes, uma vez que a contaminação nas UE obrigaria o poder público a suspender temporariamente as atividades presenciais (como ocorreu), comprometendo a frequência dos estudantes em função das quarentenas periódicas. Ludvigsson (2020), por sua vez, alega que crianças menores de 12 anos seriam assintomáticas ou menos reativas ao vírus, e que a adoção rígida de protocolos de segurança seria capaz de amenizar os riscos de contágio.

Ocorre que nenhum dos poderes federativos (municipal, estadual ou federal) se dispôs a realizar testagens periódicas dos estudantes da rede pública ou assegurou bolhas sociais a fim de facilitar o rastreamento do contágio (Vargas, 2020). Tampouco adequaram o mobiliário e a infraestrutura escolar, predispondo a comunidade a riscos. Barreiras de proteção não foram instaladas em carteiras, reformas para melhorar a circulação de ar não 
foram realizadas na maioria das escolas, tampouco disponibilizados pontos de água para higienizar as mãos, conforme sinalizam os sindicatos.

Não por acaso, a reabertura escolar coincidiu com um aumento de 57\% nas internações hospitalares por covid-19 de crianças e jovens abaixo de 18 anos. E considerando que esse grupo pode ser assintomático ou apresentar sintomas leves, é possível que o número de contaminados seja ainda maior.

O início das atividades presenciais no contexto paulistano parece corroborar a tese de Gurdasani et al. (2021), de que reabrir as escolas em períodos de crise pandêmica não apenas agrava o quadro sanitário, como torna o ensino presencial pouco efetivo para combater as vulnerabilidades socioeducacionais. Menos de um mês após a reabertura, as UE foram obrigadas a interromper $o$ atendimento presencial. E considerando que essas crianças e jovens foram submetidos a um sistema de rodízio que restringia a frequência a $35 \%$, é possível afirmar que cada estudante frequentou a escola menos de cinco vezes em 14 dias.

As eventuais externalidades negativas deflagradas pela maior circulação de pessoas na cidade - e que afetam sobretudo os mais vulneráveis - associadas aos questionáveis benefícios advindos das atividades educacionais presenciais nesse cenário de descontrole sanitário deveriam ser consideradas nas decisões relacionadas às políticas públicas educacionais.

A adoção de protocolos sanitários rigorosos, tanto no trajeto quanto no ambiente escolar, mitigaria alguns desses riscos (Gurdasani et al., 2021; Unicef, 2020). No que concerne ao trajeto, a recomendação é manter uma mesma "bolha social", ou seja, manter a coesão das pessoas que compartilham os mesmos meios de transporte. Ademais, abrir as janelas dos veículos e evitar aglomerações em espaços de circulação, pontos de embarque e desembarque e em áreas de acesso às UE (Gurdasani et al., 2021).

No ambiente escolar, recomenda-se restringir o número de pessoas, manter a homogeneidade do grupo (bolha social) e evitar a circulação em áreas comuns. Espaços amplos e abertos para ministrar as classes, como quadras e parques, a contratação de profissionais em número suficiente para assegurar a higiene local, bem como o número reduzido de estudantes por turma seriam medidas importantes para tentar conter a doença (Gurdasani et al., 2021). Ocorre que apenas $24 \%$ das unidades de educação infantil possuem equipamentos como quadras ou espaços de lazer para acomodar as crianças. E embora as unidades de ensino fundamental e médio registrem números superiores, não é possível saber as condições dessas áreas: se são espaços cobertos e se é possível ministrar classes. Tampouco se possuem dimensão suficiente para acomodar 35\% dos estudantes.

Monitores de qualidade do ar capazes de mensurar a concentração de material particulado ambiente, identificado como um possível transmissor de SarsCov-2, além da exclusão de atividades como canto, aulas com instrumentos de sopro, atividade física extenuante ou contato físico, além de testagem em massa e isolamento imediato da bolha social mediante sintomas também são imprescindíveis para evitar a contaminação entre escolares (Gurdasani et al., 2021).

Não obstante, a maior parte dessas medidas têm sido desconsideradas pelos poderes públicos. A título de exemplo, milhares de testes rápidos do governo federal perderam a validade durante o período mais crítico da sindemia, quando o número de óbitos (1.589 pessoas abaixo de 59 anos) atingiu o maior valor da série histórica no estado de São Paulo (Vargas, 2020), suscitando dúvidas sobre a competência das entidades federativas no controle das infecções por Sars-Cov-2.

Conforme evidenciado, o pico de internações por Sars-Cov-2 ocorreu 15 dias após o retorno às atividades escolares presenciais, ao passo que o decréscimo, embora discreto (8\%), também coincidiu com a interrupção das aulas, 15 dias após o fechamento das UE. Outrossim, é possível afirmar que as atividades escolares presenciais exerceram algum efeito no comportamento da sindemia, embora seja difícil dimensionar sua participação devido à dificuldade de acesso a informações oficiais. A contaminação entre escolares que optaram pelo ensino presencial comparado àqueles que permaneceram em atividade remota poderia trazer a lume os efeitos da retomada das aulas nas UE para a disseminação do vírus. Todavia, os poderes públicos mantêm os dados sob sigilo.

No que concerne aos profissionais de educação, registou-se um maior número de casos entre 
aqueles que atuam em UE situadas em territórios com menor IDHm. Alguns autores demonstram que distritos como Cidade Tiradentes, M’Boi Mirim e Sapopemba apresentam maior densidade urbana, pior qualidade construtiva (Kowarick, 1980; Marques, 2015), dificuldade de acesso à oportunidades (Giannotti et al., 2021), piores condições de trabalho (invariavelmente informais) e renda (Marques, 2015). Ademais, seus habitantes usualmente realizam viagens mais longas e de maior duração, majoritariamente por transportes públicos de massa (Marino et al., 2020, Giannotti et al., 2021). Tal condição tem sido identificada como de risco sanitário, devido à superlotação e às condições precárias de higiene nos transportes urbanos (Gutiérrez et al., 2020).

Érazoável esperar, portanto, que os profissionais de educação que atuam em equipamentos públicos situados nesses distritos (com menor IDHm) também sejam os mais expostos ao vírus, uma vez que seus habitantes apresentam maior risco de contágio. Com efeito, aspectos socioeconômicos e territoriais parecem agravar os efeitos sanitários da covid-19, inclusive nas escolas inseridas nesses territórios, reproduzindo as desigualdades sanitárias extramuros também no âmbito escolar (Ahmed et al., 2020; Horton, 2020; Kirby, 2020).

O desinvestimento público em educação, consolidado desde 2017 pela lei orçamentária que estabeleceu um teto para os gastos obrigatórios da União (Mariano, 2017) - e que atingiu o menor orçamento da década em 2020 - também pode ter influenciado na transmissão de covid-19 entre os membros das UE, uma vez que a escassez de recursos pode ter comprometido a readequação dessas unidades, bem como a contratação de equipes de apoio e limpeza em número suficiente, além do cumprimento de protocolos sanitários obrigatórios, tais como espaços arejados para ministrar as aulas. Um estudo recente que acompanhou quase 13 mil profissionais de educação identificou uma incidência três vezes maior de contaminação entre professores de educação básica comparado à população adulta (19 a 59 anos) (Repu, 2021).

Os resultados do presente artigo, embora não possibilitem elaborar tais conclusões, permitem afirmar que profissionais de UE localizadas em distritos com menor IDHm evidenciaram maiores taxas de contaminação por covid-19, com destaque para Cidade Tiradentes. Em conjunto, é possível afirmar que o retorno presencial às UE não é seguro enquanto as taxas de contaminação por covid-19 permanecerem elevadas, sobretudo nos territórios mais vulneráveis do município de São Paulo.

\section{Considerações finais}

0 retorno às aulas presenciais coincidiu com o pico no número de internações e óbitos por covid-19 entre crianças e jovens. As razões para esse cenário incluem sucateamento das escolas públicas, infraestrutura e mobiliário escolar precário, falta de equipe de apoio e limpeza para garantir a higienização dos equipamentos educacionais, ausência de testagem de estudantes e profissionais de ensino, bem como dificuldade em observar os protocolos sanitários devido aos baixos investimentos públicos em educação, que no ano de 2020 registrou o menor valor da década.

O histórico processo de espoliação urbana que compromete a qualidade territorial e dificulta o acesso à oportunidades também engendra efeitos sobre a contaminação de covid-19, que resvalam sobretudo nos profissionais das UE localizadas nesses territórios espoliados, reproduzindo as desigualdades sociossanitárias também no âmbito escolar.

Portanto, retomar as atividades presenciais escolares sem mitigar os agravantes que aumentam o risco de infecção por Sars-Cov-2 pode ensejar um aumento de internações e eventuais óbitos entre estudantes e profissionais de ensino, principalmente naqueles que atuam em UE inseridas em áreas com menor qualidade socioterritorial, agravando uma sindemia que somente no Brasil vitimou 560 mil vidas.

\section{Referências}

AHMED, F. et al. Why inequality could spread COVID-19, The Lancet Public Health, Londres, v. 5, n. 5, p. e240, 2020. DOI: 10.1016/S2468-2667(20)30085-2

CMSP - COMPANHIA DO METROPOLITANO DE SÃO PAULO. Pesquisa Origem-Destino (2017).

São Paulo, 2019. Disponível em: <http://www.metro. sp.gov.br/pesquisa-od/>. Acesso em: 22 mar. 2021. 
COMISSÃO DE BIOSSEGURANÇA do CCAE Centro de Ciências Aplicadas e Educação. Protocolo de biossegurança e diretrizes gerais para o ccae pandemia do coronavírus (covid-19), Mamanguape: UFPB, 2020. Disponível em: <http://www.ccae.ufpb.br/ccae/contents/ documentos/documentos-institucionais/direcao/ protocolo_de_biossegurana_e_diretrizes_gerais_--_ ccae_-_verso_aprovada_pelo_co.pdf $>$.

Acesso em: 22 mar. 2021.

\section{DIESE - DEPARTAMENTO INTERSINDICAL DE} ESTATÍSTICAS E ESTUDOS SOCIOECONÔMICOS.

Índice do custo de vida. [S.l.], 2020. Disponível em: <https://www.dieese.org.br/analiseicv/icv.html>. Acesso em: 22 mar. 2021.

FIGUEIREDO, P.; IANELLI, C. Volta às aulas é adiada em 530 escolas e creches municipais de São Paulo por falta de funcionários de limpeza, Globo G1, São Paulo, fev. 2021. Disponível em: <https://g1.globo.com/sp/sao-paulo/ noticia/2021/o2/12/volta-as-aulas-e-adiada-emescolas-e-creches-municipais-de-sao-paulo-porfalta-de-funcionarios-de-limpeza-sindicatoestima-em-70o.ghtml>. Acesso em: 22 mar. 2021.

GIANNOTTI, M. et al. Inequalities in transit accessibility: Contributions from a comparative study between Global South and North metropolitan regions. Cities, v. 109, 2021. DOI: 10.1016/j.cities.2020.103016

GURDASANI, D. et al. School reopening without robust COVID-19 mitigation risks accelerating the pandemic, The Lancet, London, v. 397, n. 10280, p. 1177-1178, 2021. DOI: 10.1016/So140-6736(21)oo622-X

GUTIÉRREZ, A., MIRAVET, D., DOMÈNECH, A. COVID-19 and urban public transport services: emerging challenges and research agenda, Cities \& Health, Abingdon, p. 1-4, ago. 2020. DOI: 10.1080/23748834.2020.1804291

HOCHMAN, G., ARRETCHE, M., MARQUES, E. (Org.) Políticas públicas no Brasil. Rio de Janeiro:

Editora Fiocruz, 2007.

HORTON, R. Offline: COVID-19 is not a pandemic, The Lancet, London, v. 396, n. 10255, p. 874, 2020. DOI: $10.1016 /$ So140-6736(20)32000-6
IBARRA-ESPINOSA, S. et al. Negative-binomial and quase-poisson regressions between COVID-19, mobility and environment in São Paulo, Brazil, MedRxiv, New York, fev. 2021. DOI: 10.1101/2021.02.08.21250113

IBGE - Instituto Brasileiro de Geografia e Estatística. Censo Escolar: sinopse. [S.l.], 2021. Disponível em: <https://cidades.ibge.gov.br/ brasil/sp/sao-paulo/pesquisa/13/5908>.

Acesso em: 22 mar. 2021.

INEP - Instituto Nacional de Estudos e Pesqisas Educacionais Anísio Teixeira. Censo Escolar 2020. $Q E d u, 2021$ (c). Disponível em: <https://qedu. org.br/estado/125-sao-paulo/censo-escolar?yea $\mathrm{r}=2020 \&$ dependence=o\&localization=o\&educati on_stage=o\&item=>. Acesso em: 4 ago. 2021.

KIRBY, T. Evidence mounts on the disproportionate effect of COVID-19 on ethnic minorities, The Lancet. Respiratory Medicine, London, v. 8, n. 6, p. 547-548, 2020. DOI: $10.1016 /$ S2213-2600(20)30228-9

KOWARICK, L. A espoliação urbana. São Paulo: Paz e Terra, 1980.

LUDVIGSSON, J. F. Systematic review of COVID-19 in children shows milder cases and a better prognosis than adults, Acta Paediatrica, v. 109, n. 6, p. 1088-1095, 2020. DOI: 10.1111/apa.15270

MARIANO, C. M. Emenda constitucional 95/2016 e o teto dos gastos públicos: Brasil de volta ao estado de exceção econômico e ao capitalismo do desastre, Revista de Investigações Constitucionais, Curitiba, v. 4, n. 1, p. 259-281, 2017. DOI: $10.5380 /$ rinc.v411.50289

MARINO, A. et al. Circulação para trabalho explica concentração de casos de Covid-19: [S.l.]: Labcidade, 2020. Disponível em: <http://www.labcidade.fau.usp.br/ circulacao-para-trabalho-inclusive-servicosessenciais-explica-concentracao-de-casos-decovid-19/>. Acesso em: 22 mar. 2021.

MARQUES, E. (Org.) A metrópole de São Paulo no século $X X I$ : espaços, heterogeneidades e desigualdades, São Paulo: Editora Unesp, 2015.

MARTINS, L., BRAGANÇA, R., BRITO, A. Secretário de São Paulo pressiona municípios por 
volta às aulas: "estamos prontos”, Uol, São Paulo, 2021. Disponível em: <https://educacao.uol.com. br/noticias/2021/o1/13/anuncio-educacao-13-01. htm>. Acesso em: 22 mar. 2021.

\section{REPU - REDE ESCOLA PÚBLICA E} UNIVERSIDADE. Monitoramento de casos de Covid-19 na rede estadual de São Paulo [Nota Técnica]. São Paulo, 2021. Disponível em: <https://3c6oc040-0201-4188-bfd9-ddc2o8c6ad1a. filesusr.com/ugd/9cce3o_232a4b26e21c4a6oa75o 731ec5a27cdd.pdf>. Acesso em: 22 mar. 2021.

SÃO PAULO. Instrução normativa SME N ${ }^{\circ} 58$, de 18 de dezembro de 2020. Dispõe sobre a organização das unida-des de educação infantil, de ensino fun-damental, de ensino fundamental e médio e dos centros educacionais unificados da rede municipal de ensino para o ano de 2021. Diário Oficial [da] Cidade de São Paulo. São Paulo, n. 240, p. 14, 2020. Disponível em: http://www.docidadesp.imprensaoficial.com.br/ NavegaEdicao.aspx?ClipID=2e38f 54 ceedaze $86 \mathrm{fb} 4$ agc8bde2ff4d3\&PalavraChave=Aulas+presenciais. Acesso em: 22 mar. 2021.

SME - SECRETARIA MUNICIPAL DE EDUCAÇÃO. Transporte Escolar Gratuito - TEG. SME Portal Institucional. São Paulo, 2021. Disponível em: <https://educacao.sme.prefeitura.sp.gov.br/ transporte-escolar-gratuito/>.

Acesso em: 22 mar. 2021.

\section{SINPEEM - SINDICATO DOS PROFISSIONAIS} EM EDUCAÇÃO NO ENSINO MUNICIPAL - SP. Atualização dos casos de contaminação na rede informados ao sinpeem - 05/o3/2021. SINPEEM, São Paulo, 2021. Disponível em: <https://www. sinpeem.com.br/sites/arquivos/downloads/ balancocovid-o5032021-valeeste.pdf>.

Acesso em: 22 mar. 2021.

TODOS PELA EDUCAÇÃO. Execução orçamentária do ministério da educação (MEC). $6^{\circ}$ Relatório Bimestral. São Paulo, 2021. Disponível em: <https://todospelaeducacao.org.br/wordpress/wpcontent/uploads/2021/02/6 ${ }^{\circ}$-Relatorio-Bimestralda-Execucao-Orcamentaria-do-MEC.pdf $>$.

Acesso em: 22 mar. 2021.

\section{UNICEF - UNITED NATIONS CHILDREN'S} FUND. New guidelines provide roadmap for safe reopening of schools, UNICEF, New York, 2020. Disponível em: <https://www.unicef.org/pressreleases/new-guidelines-provide-roadmap-safereopening-schools>. Acesso em: 22 mar. 2021.

VAN LANCKER, W., PAROLIN, Z. COVID-19, school closures, and child poverty: a social crisis in the making, The Lancet Public Health, London, v. 5 , n. 5, p. e243-e244, 2020. DOI: 10.1016/S24682667(20)30084-0

VARGAS, M. Prazo de validade pode levar governo federal a jogar fora 6,8 milhões de testes. O Estado de São Paulo, São Paulo, 22 nov. 2020. Disponível em: <https://saude.estadao.com.br/ noticias/geral,prazo-de-validade-pode-levargoverno-federal-a-jogar-fora-6-8-milhoes-detestes,70003523522>. Acesso em: 14 out. 2021.

VIRGINIO, A. S. Educação, Desigualdade e COVID-19, Notícias IFCH - UFRGS, Porto Alegre, 2021. Disponível em: <https://www.ufrgs.br/ifch/ index.php/br/educacao-desigualdade-e-covid-19>. Acesso em: 22 mar. 2021.

ZHOU, F. et al. Clinical course and risk factors for mortality of adult inpatients with COVID-19 in Wuhan, China: a retrospective cohort study, The Lancet, London, v. 395, n. 10229, p. 1054-1062, 2020. DOI: 10.1016/So140-6736(20)30566-3

\section{Agradecimentos}

Costaria de agradecer à Flávia Cristina Souza Silva pela tabulação e organização dos dados disponibilizados pelo Sinpeem.

\section{Contribuição dos autores}

Rumenig foi responsável pela concepção do estudo, obtenção, análise e interpretação dos dados, elaboração e revisão do manuscrito e aprovação da versão final para publicação. Por conseguinte, assume total responsabilidade pela exatidão $e$ integridade da pesquisa.

Recebido: 05/08/2021

Reapresentado: 05/08/2021

Aprovado: 27/09/2021 